\title{
Effects of pretraining on conditioning-enhanced neophobia: Evidence for separable mechanisms of neophobia and aversion conditioning
}

\author{
JOSEPH J. FRANCHINA and DAVID W. GILLEY \\ Virginia Polytechnic Institute and State University, Blacksburg, Virginia
}

\begin{abstract}
In two experiments, rats $(n=228)$ received pretraining access to a distinctive novel flavor (saline) followed by aversion conditioning to a different novel conditioned stimulus (CS) (saccharin). Then the rats were tested for aversion to the CS (saccharin) or for conditioning-enhanced neophobia to a third novel flavor (casein hydrolysate). Pretraining access to a distinctive novel flavor that differed from the CS reliably reduced the magnitude of conditioning-enhanced neophobia to casein, but did not reliably affect conditioned aversion effects to the CS. Pretraining access to the CS reduced aversion effects to the CS and reduced postconditioning neophobia to casein to the performance level shown by ingestion-toxin controls. Results were consistent with the view (Braveman \& Jarvis, 1978) that conditioned aversion and neophobia may be independent phenomena with separable underlying mechanisms.
\end{abstract}

Neophobia refers to the natural tendency of omnivores, such as rats, initially to avoid ingesting substantial amounts of unfamiliar/novel edibles (Barnett \& Cowan, 1976). When ingestion of a novel flavor is followed by toxicosis, conditioned taste aversion (CTA) occurs and avoidance behavior subsequently persists to that novel flavor. Studies of flavor preexposure have shown that prior access to the test flavor reliably reduces neophobia and CTA (e.g., Domjan, 1976; Siegel, 1974). Consequently, neophobia and CTA have been viewed as being related to each other, possibly having mechanisms in common (e.g., Barnett \& Cowan, 1976; Domjan, 1980; Krane, Sinnamon, \& Thomas, 1976; Mitchell, Winter, \& Moffitt, 1980; Tarpy \& McIntosh, 1977).

However, Braveman and Jarvis (1978) and, with some qualifications, Miller and Holzman (1981, p. 99) have argued for the separability of neophobia and CTA. Braveman and Jarvis showed that prior access to a distinctive novel flavor reduced neophobia, but not CTA, to another novel flavor. For CTA, only prior access to the flavor conditioned stimulus (CS) reduced the aversion. These results suggested that for neophobia, preexposure reduced the aversive properties of novelty (Domjan, 1976) through a general process that is not necessarily related to specific flavor characteristics, such as taste modality, intensity, or palatability (Best \& Batson, 1977; Braveman \& Jarvis, 1978; Miller \& Holzman, 1981). For CTA, conditioning presumably occurs to specific flavor characteristics. Thus, preexposure to a novel flavor whose characteristics (e.g.,

\footnotetext{
These data were reported at the Midwestern Psychological Association Meeting, Detroit, May 1981. Thanks are due to A. B. Dyer for critically reading this paper. Requests for reprints should be sent to the first author at the Department of Psychology, Virginia Polytechnic Institute and State University, Blacksburg, VA 24061.
}

taste) differ from those of the CS may not affect CTA because of the specificity of conditioning to the CS.

In previous studies of the separability of neophobia and CTA, the effects of preexposure on neophobia were measured before the measurement of preexposure's effects on CTA (e.g., Braveman \& Jarvis, 1978). This order of measurement would have permitted neophobia effects to influence CTA (although they apparently did not) but precluded the possible influence of CTA effects on neophobia. Thus, evidence for the separability of neophobia and CTA may have been due to the testing of neophobia before CTA. If neophobia and CTA are separable from each other, evidence for separability should be obtainable whether neophobia is tested before (e.g., Braveman \& Jarvis, 1978) or after the acquisition of CTA.

Prior acquisition of CTA to a novel flavor subsequently yields an increase in neophobia to other novel flavors (Best \& Batson, 1977; Carroll, Dinc, Levy, \& Smith. 1975; Franchina \& Fitzgerald, 1983). This postconditioning enhancement of neophobia presumably reflects the generalization of aversion from the original novel CS to other novel flavors (Domjan, 1980). Because postconditioning neophobia effects follow and depend upon prior acquisition of CTA (Franchina \& Fitzgerald, 1983), evidence for the separability of neophobia and CTA in this context would complement earlier findings (e.g., Braveman \& Jarvis, 1978). Such evidence would consist in a finding that prior access to a novel flavor did not retard CTA to another novel flavor but did reduce the subsequent demonstration of increased neophobia to a third novel flavor. Preconditioning access to a distinctive novel flavor that differs from the novel CS should not reduce CTA (relative to that in controls) because of the specificity of aversion conditioning. However, preconditioning access to a distinctive novel flavor may reduce the aversiveness of 
novelty as a general process, separate from flavor-specific stimuli such as taste or intensity. If so, one basis for the postconditioning enhancement of neophobia would be attenuated, and thus the demonstration of postconditioning neophobia would be reduced relative to that for nonpreexposure controls. To investigate this possibility, we gave rats preconditioning access to a novel flavor (saline) followed by CTA to another novel flavor (saccharin) and then neophobia testing to a third novel flavor (casein hydrolysate). Evidence for the separability of neophobia from CTA would consist in the finding that prior access to saline did not reduce CTA to saccharin, but did reduce postconditioning neophobia to casein, all relative to waterpreexposure controls. We performed four experiments, Experiments $1 \mathrm{a}, 1 \mathrm{~b}, 2 \mathrm{a}$, and $2 \mathrm{~b}$, using the same general procedures.

\section{GENERAL METHOD}

\section{Subjects}

Each experiment used experimentally naive male albino rats (Rattus norvegicus), 90 to 100 days old, from the colony maintained by the Department of Psychology at Virginia Polytechnic Institute and State University. On Day 0 each rat was housed in a single hanging cage in a room, $2.4 \times 2.4 \times 3.0 \mathrm{~m}$. Room temperature was $24^{\circ} \pm 1^{\circ} \mathrm{C}$; illumination level was $240 \mathrm{~lx}$. The light/dark cycle was $14 \mathrm{~h} / 10 \mathrm{~h}$, with light onset at 08:00 h. Food (Wayne Lab Blox) was available ad lib except as noted. Daily fluid access occurred at 08:30 h and 15:30 h.

\section{Procedure}

Experiments 1a and $1 \mathrm{~b}$ consisted of 5 days for habituation to fluid deprivation and preexposure procedures (Days 1-5), 2 days for aversion conditioning, 1 day for recovery from toxicosis, and 3 days of testing for CTA or neophobia. Experiments $2 a$ and $2 b$ followed the same sequence but with only 1 conditioning day.

In all experiments, on Day 1 the rats received 15 min access to tap water and then were randomly assigned to preexposure conditions. On Days 2 to 5 the rats received $10 \mathrm{~min}$ access to saline, saccharin, or deionized distilled water twice a day.

Specific aversion conditioning procedures for each experiment will be described later. However, in all experiments the toxin was $.15 \mathrm{M}$ lithium chloride $(\mathrm{LiCl})$, injected intraperitoneally at $2.0 \%$ of the rat's body weight within $10 \mathrm{~min}$ after flavor access. After injection the rat was returned to its cage, where it received distilled water for $10 \mathrm{~min}$ at $15: 30 \mathrm{~h}$. On the day following the completion of conditioning (recovery day), the rats received $10 \mathrm{~min}$ access to distilled water at $08: 30 \mathrm{~h}$ and $15: 30 \mathrm{~h}$.

Testing began at 08:30 h on the day after recovery, and consisted of one test trial per day for 3 days. Each trial provided 10 min access to a single bottle that contained the appropriate flavor. The performance measure was amount drunk, calculated, to the nearest $100 \mathrm{mg}$, as the difference between pre- and posttrial bottle weights. On each test day each rat received 10 min access to distilled water at $15: 30 \mathrm{~h}$.

\section{EXPERIMENTS 1a AND 1b}

\section{Method}

\section{Experiment 1a: CTA}

Forty-eight rats were randomly assigned to a $3 \times 2$ design $(n=8)$ : three preexposure fluids $(0.9 \%$ saline $[\mathrm{N}], 0.25 \%$ saccharin [S], and distilled water [W]) and two flavor CSs (saline and saccharin) in aversion conditioning and testing. Groups NN, SN, and $\mathrm{WN}$ received pretraining with saline, saccharin, and distilled water (first letter), respectively, and then aversion conditioning and testing with saline (second letter). Groups SS, NS, and WS received pretraining with saccharin, saline, and water, respectively, and conditioning and testing with saccharin. Aversion conditioning occurred on Days 6 and 7. On each day each rat received 5 min access to saline or saccharin followed by an injection of $\mathrm{LiCl}$. After the recovery day, the rats were tested for aversion to the original CS, saline or saccharin, on Days 9-11.

\section{Experiment 1b: Neophobia}

Seventy-two rats were randomly assigned to a $3 \times 3$ design $(n=8)$ that orthogonally combined $0.9 \%$ saline $(N), 0.25 \%$ saccharin (S), and distilled water (W) across pretraining and conditioning. Groups NN, SN, and WN and Groups SS, NS, and WS in this design were treated like those in Experiment 1a. Experiment $1 \mathrm{~b}$ also included Groups NW, SW, and WW, ingestion-toxin control groups that received pretraining with saline, saccharin, or distilled water, followed by distilled water- $\mathrm{LiCl}$ pairings. After the recovery day all groups were tested for neophobia to $3 \%$ casein hydrolysate.

Flavor solutions were mixed (w/v), using deionized distilled water, approximately $18 \mathrm{~h}$ before the next access period. For the saline solution, we used reagent grade sodium chloride; for the saccharin solution, we used commercial grade sodium saccharin; for the casein solution, we used casein hydrolysate (enzymatic, extrasoluble) from ICBN Biochemical Company. Statistical evaluation was by analysis of variance (ANOVA) for repeated measures, one-way ANOVA, $t$ tests, and Scheffé tests as appropriate (aipha level $=0.05$ )

\section{Experiment 1a: CTA}

Pretraining and injection-day intakes. In the morning access period of the last pretraining day, Day 5, mean intakes of saline, saccharin, and distilled water were $14.3 \mathrm{~g}, 12.6 \mathrm{~g}$, and $12.2 \mathrm{~g}$, respectively $(F<1)$. Table 1 shows mean intakes of saline and saccharin CSs on each injection day. Intakes on Injection Day 2 show the effects of the flavor-toxin pairing on Injection Day 1. Pretraining access to the CS (Pre saline for saline CS and Pre saccharin for saccharin CS) yielded negligible changes in intake over days. Pretraining access to distilled water or

Table 1

Mean Intakes (in Grams) of Saline and Saccharin on Injection Days 1 and 2 for Groups Preexposed to Saline, Saccharin, or Distilled Water in Experiment 1a and Mean Intakes (in Grams) for Saline, Saccharin, and Distilled Water on Injection Days 1 and 2 for Groups Preexposed to Saline, Saccharin, or Water in Experiment 1b

\begin{tabular}{|c|c|c|c|c|c|c|}
\hline \multirow{2}{*}{$\begin{array}{l}\text { Group } \\
\text { Preexposed }\end{array}$} & \multicolumn{2}{|c|}{ Saline CS } & \multicolumn{2}{|c|}{ Saccharin CS } & \multicolumn{2}{|c|}{ Water } \\
\hline & Day 1 & Day 2 & Day 1 & Day 2 & Day 1 & Day 2 \\
\hline \multicolumn{7}{|c|}{ Experiment la } \\
\hline Saline & 7.4 & 6.0 & $5.9^{*}$ & $1.6^{*}$ & & \\
\hline Saccharin & $6.5^{*}$ & $2.6^{*}$ & 7.4 & 5.6 & & \\
\hline Water & $6.1^{*}$ & $1.4^{*}$ & $5.1^{*}$ & $1.3 *$ & & \\
\hline \multicolumn{7}{|c|}{ Experiment $\mathbf{l b}$} \\
\hline Saline & 6.3 & 6.8 & $5.0^{*}$ & $1.7 *$ & 7.0 & 7.1 \\
\hline Saccharin & $8.1^{*}$ & $3.2 *$ & 7.7 & 6.6 & 6.8 & 6.0 \\
\hline Water & $6.7^{*}$ & $2.4^{*}$ & $6.3^{*}$ & $1.5^{*}$ & 8.33 & 7.1 \\
\hline
\end{tabular}

*Day 1 versus Day $2, p<.01$. 
to a novel flavor that differed from the CS (Pre saccharin for saline CS and Pre saline for saccharin CS) reliably decreased intake over days ( $p s<.01)$.

Saline and saccharin aversion. Figure 1 presents mean intakes of saline and saccharin CSs in testing. For testing with saline, pretraining access to saccharin or to distilled water yielded similar mean saline intakes, which were below the mean intake for pretraining with saline. For testing with saccharin, pretraining with saline or distilled water yielded similar mean saccharin intakes, which were below the mean intake for pretraining with saccharin. An ANOVA of the intakes of saline and saccharin, separately, yielded reliable effects for pretraining fluid, for test trials, and for the interaction of the two [for saline, $F(2,21)=$ $34.19, F(2,42)=34.65$, and $F(4,42)=8.24$, respectively, all $p$ s $<.001$; for saccharin, $F(2,21)=60.60$, $F(2,42)=26.46$, and $F(4,42)=6.08$, respectively, all $p s<.001]$. For saline intakes, groups preexposed to $S$ or to $\mathrm{W}$ each differed reliably $(p<.001)$ from the group preexposed to $\mathbf{N}$ but not from each other $(t<1)$. For saccharin intakes, groups preexposed to $\mathrm{N}$ or to $\mathrm{W}$ each differed reliably $(p<.001)$ from the group preexposed to $S$ but not from each other $(t<1)$. Together with the injection-day data, these results provided reliable evidence of aversion to novel saline and saccharin CSs. For each CS, pretraining with a distinctive novel flavor that differed from the novel CS and pretraining with distilled water yielded apparently similar CTA effects.

\section{Experiment 1b: Neophobia}

Pretraining and injection-day intakes. Mean intakes of saline, saccharin, and distilled water on the morning of pretraining Day 5 were $13.8 \mathrm{~g}, 12.6 \mathrm{~g}$, and $11.6 \mathrm{~g}$, respectively $(F<1)$. Table 1 shows mean intakes of saline, saccharin, and distilled water on each injection day of Experiment 1b. Results for saline and saccharin CSs were similar to those for like groups in Experiment 1a.

Postconditioning neophobia. Figure 1 shows mean intakes of casein for each preexposure and CS condition. Test Trial 1 shows less casein intake for groups conditioned with novel saline or saccharin CSs than for groups trained with a familiarized flavor or distilled water. Specifically, Groups SN and WN appear to have consumed less casein than did Groups NN or NW; Groups NS and WS appear to have consumed less casein than did Groups SS or SW. More importantly, for novel CS groups, preconditioning access to a novel flavor that differed from the CS yielded higher casein intake than did precondition-

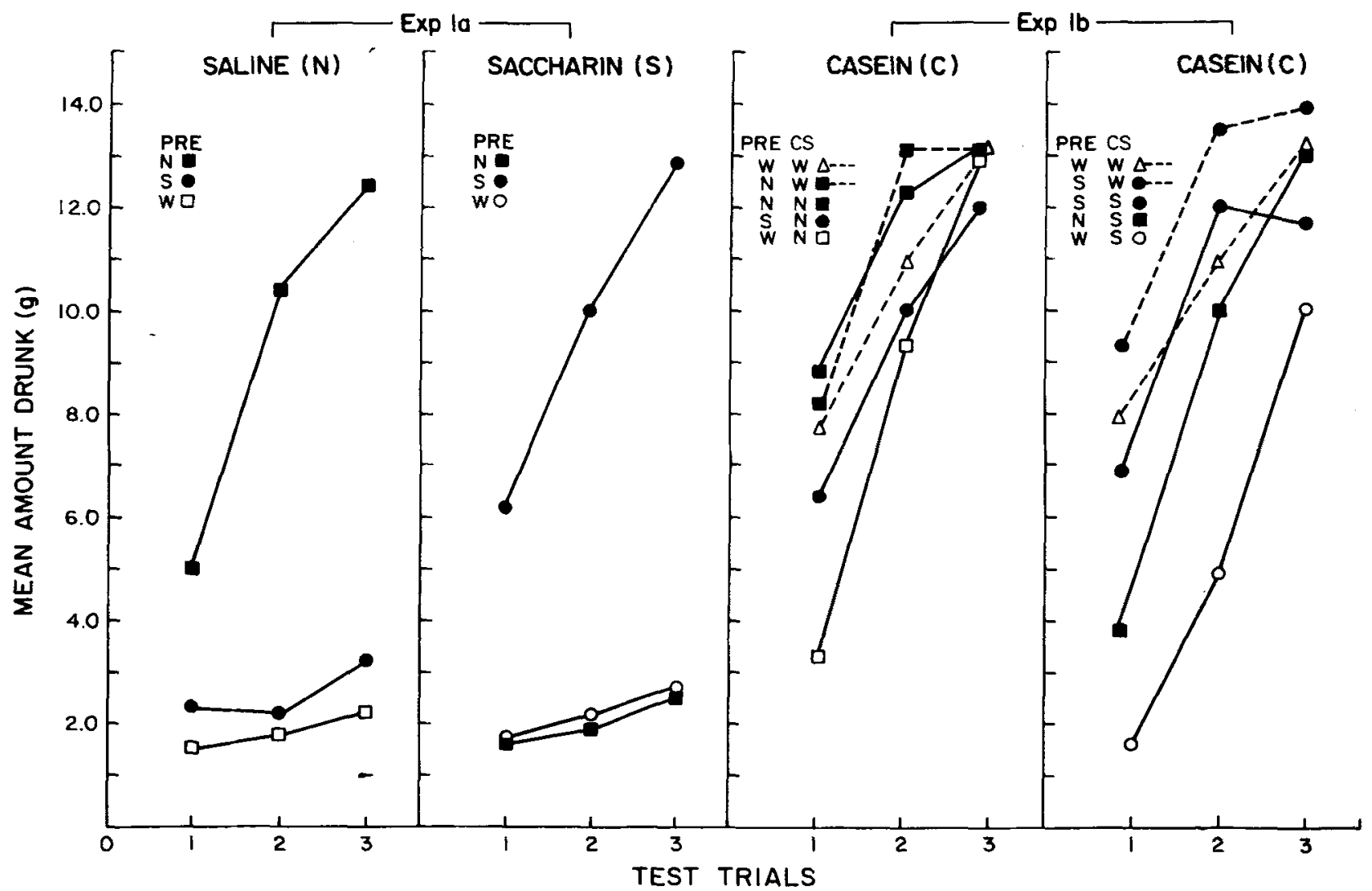

Figure 1. For Experiment (Exp) 1a: Mean amount (in grams) of saline (N) or saccharin (S) drunk on Test Trials 1-3 after pretraining (PRE) access to saline (N), saccharin (S), or distilled water (W) and aversion training with saline or saccharin CSs. For Experiment (Exp) 1b: Mean amount (in grams) of casein (C) drunk on Test Trials 1-3 after pretraining (PRE) access to saline (N), saccharin (S), or distilled water (W) and aversion training (CS) with saline, saccharin, or distilled water. The data of ingestion-toxin controls, Group WW, are presented in both casein (C) sections for ease of comparison. 
ing access to distilled water. Group SN drank more casein than did Group WN; Group NS drank more casein than did Group WS.

An ANOVA of Trial 1 data for Groups SN, WN, and NN and for Groups NS, WS, and SS, separately, yielded a reliable effect for pretraining fluid in each case $[F(2,21)$ $=10.09$ and 9.77 , respectively, $p<.001]$. Scheffé comparisons showed that Group SN differed reliably from Group WN but not from Group NN (despite apparent differences shown in Figure 1). Groups WN and NN differed reliably. Scheffé comparisons of Groups WS, NS, and SS revealed reliable differences in all cases. Performance of Groups NN and SS did not differ reliably from that of ingestion-toxin controls (Groups NW and SW, respectively). To compare performance of Groups NW and SW with that of Group WW, an ANOVA was applied to their casein intakes on Trial 1. No reliable groups effect was revealed here (or in the ANOVA for these groups over all test trials).

Thus, on Trial 1, neophobia to casein was reliably greater after conditioning with a novel CS than after training with a familiarized CS or after ingestion-toxin control procedures. For novel-CS conditioning groups, preconditioning access to a novel flavor that differed from the CS reliably reduced the magnitude of conditioningenhanced neophobia relative to that for prior access to water.

Trial 2 results appear to be similar to those of Trial 1. Trial 2 shows less casein intake for Groups WN and SN than for Groups NN and NW, and less casein intake for Groups WS and NS than for Groups SS and SW. Of the novel-CS groups, Group SN drank more than did Group WN, and Group NS drank more than did Group WS. However, ANOVA yielded a reliable effect of pretraining fluid for Groups NS, WS, and SS $[F(2,21)$ $=21.69, p<.001]$ but not for Groups SN, WN, and NN.

The appropriateness of the preceding single-trial analyses was substantiated by ANOVA over all the data for Groups SN, WN, and NN and for Groups NS, WS, and SS, separately, which yielded a reliable pretraining fluid $\times$ test trial interaction in each case $[F(4,42)=2.95$ and $4.29, p<.03$ and .005 , respectively].

\section{Discussion}

Experiments 1a and $1 \mathrm{~b}$, together, provide some evidence for the separability of neophobia and CTA. Preconditioning access to a novel flavor that differed from the novel CS did not retard CTA relative to that shown by water-preexposed controls in Experiment 1a, but reliably reduced the demonstration of enhanced neophobia following conditioning in Experiment $1 \mathrm{~b}$. Thus, preexposure procedures that apparently did not affect CTA did reliably affect subsequent neophobia.

Specifically, in Experiment $1 \mathrm{~b}$, reliable evidence of conditioning-enhanced neophobia was that casein intake was reliably less for Groups SN, WN, NS, and WS than for Group WW. Group WW had received distilled water- toxin pairings prior to testing with novel casein. Thus, performance by this group (and, by implication, Groups NW and SW, which had also received distilled watertoxin pairings) indicates the effects of the ingestion-toxin contingency on subsequent responding to novel casein. The lower intakes of Groups SN, WN, NS, and WS, relative to Groups WW, NW, and SW, then, indicate the effects of novel CS-toxin pairings (conditioning procedures) on responding to novel casein. Presumably, conditioning augmented the aversive properties of novelty occasioned by novel casein, and thereby increased neophobia relative to that for ingestion-toxin controls.

However, the level of enhanced neophobia was reliably less for Groups SN and NS than for Groups WN and WS, at least on Test Trial 1. These data suggest that preconditioning access to a novel flavor that differed from the CS yielded effects that persisted through the acquisition of CTA and reliably reduced the magnitude of postconditioning neophobia. Because Groups SN and NS both showed a reliable reduction in postconditioning neophobia, it appears that the effects of preexposure were not flavor-specific. These data from Groups SN and NS, together with the results of Experiment 1a, suggest that preexposure affected neophobia through a general process related to novelty, but affected CTA on the basis of specific flavor characteristics.

Unfortunately, the preceding conclusion is tentative, because of the possible occurrence of floor effects in CTA and the suggestion of flavor-specific effects in postconditioning neophobia. First, in Experiment 1a, the conditioning procedure of two flavor-toxin pairings may have yielded a floor effect which may have obscured differences in CTA performance between water-preexposed groups and groups preexposed to a novel flavor (not the CS). None of these preexposed groups showed a reliable change in performance over Trials 1-3 of CTA testing, suggesting that training procedures strongly suppressed ingestion of the novel CS. Second, in Experiment $1 b$, some results for postconditioning neophobia may have reflected differences which were attributable to specific properties of the flavors used. For example, on Trial 1, Groups SN and NS showed a reduction in postconditioning neophobia relative to Groups WN and WS, but the reduction was greater for Group SN. A post hoc ANOVA over all test trials further revealed that postconditioning neophobia was reliably greater for Group WS, which was conditioned to novel saccharin, than for Group WN, which was conditioned with novel saline. Accordingly, the difference in postconditioning neophobia between Groups SN and NS may have reflected which flavor, saline or saccharin, was the conditioning flavor and which flavor was the preexposed flavor. On Trial 2, reliable differences due to pretraining occurred for Groups NS, SS, and WS but not for Groups SN, NN, and WN. These data again suggest that flavor-specific stimuli (e.g., taste quality or intensity) may have influenced the effects of preexposure, the strength of conditioning, and/or the transfer of these effects to neophobia testing. If so, then 
the conclusion that neophobia and CTA are separable becomes tentative. Experiments $2 a$ and $2 b$ were performed to remove the possible problem of floor effects in CTA and to minimize the likelihood that the results of postconditioning neophobia were due to flavor-specific properties of the stimuli used.

\section{EXPERIMENTS 2a AND 2b}

Experiments $2 \mathrm{a}$ and $2 \mathrm{~b}$ investigated the separability of neophobia and CTA with a slightly different approach from that of Experiments 1a and $1 b$. Experiments $2 a$ and $2 b$ studied postconditioning neophobia following serial aversion conditioning, in which a familiarized (preexposed) flavor and a different novel flavor occurred in series prior to the toxin (e.g., Revusky \& Bedarf, 1967). Then CTA was tested to the familiarized CS and to the novel CS in Experiment $2 \mathrm{a}$ and postconditioning neophobia was tested to casein hydrolysate in Experiment $2 \mathbf{b}$.

Serial aversion conditioning with a familiarized and a novel CS yields reliable CTA to the novel CS and negligible effects for the familiarized CS (Ahlers \& Best, 1971; Franchina, Silber, \& May, 1981). Because these effects occur regardless of whether the familiar or the novel flavor occurs nearer in time to the toxin, they suggest that novel flavors and toxicosis are selectively associable (Revusky \& Bedarf, 1967). If this selective associability operates for postconditioning neophobia, then, even with a familiarized CS present in the training sequence, serial aversion conditioning should yield reliable enhancement of neophobia relative to that for ingestion-toxin controls.

However, according to Braveman and Jarvis (1978), preexposure to a distinctive novel flavor should reduce the aversiveness of novelty, and thereby curtail neophobia to other novel flavors. Consequently, the enhancement of neophobia should be less after serial conditioning for groups that had received preexposure to a novel flavor (not the novel CS) than for groups that had received water. If this were found to be the case in Experiment $2 b$, and if novel flavor preexposure and water preexposure were found to yield similar CTA effects to the novel CS in Experiment $2 a$, these findings would constitute evidence for the separability of neophobia and CTA.

In order to preclude the occurrence of a floor effect, which may have occurred in CTA in Experiment 1a, we administered only one aversion conditioning trial in Experiments $2 \mathrm{a}$ and $2 \mathrm{~b}$. Furthermore, to minimize possible differences in conditioning or preexposure effects due to the specific characteristics (e.g., taste or concentration) of saline or saccharin flavors, the distinctiveness/intensity of the saline was increased by using a $2.0 \%$ concentration. Perhaps, compared to saccharin in Experiments 1a and $1 b$, the saline flavor was a relatively weaker stimulus.

\section{Experiment 2a: CTA}

\section{Method}

Forty-eight rats received habituation procedures and pretraining access to $2.0 \%$ saline, $0.25 \%$ saccharin, or distilled water on Days 1-5, as in Experiments 1a and 1b. On Day 6 (aversion con- ditioning), each rat received in series $2 \mathrm{~min}$ of fluid access, $2 \mathrm{~min}$ rest, and then $2 \mathrm{~min}$ of fluid access. Each rat received an injection of $\mathrm{LiCl} 10 \mathrm{~min}$ after the second access period. Group NNS $(n=16)$ received pretraining with saline $(N)$ and serial conditioning with familiarized saline $(\mathrm{N})$ and novel saccharin (S). Group SSN $(n=$ 16) received pretraining with saccharin $(S)$ and conditioning with familiarized saccharin (S) and novel saline (N). Groups that received pretraining access to distilled water $(W)$ received serial conditioning with water $(W)$ and novel saline $(N)$ or with water $(W)$ and novel saccharin (S) (Groups WWN and WWS, $n=8$ each). The order of CS presentation in conditioning was counterbalanced.

Day 7 was recovery day. Days 8-10 were CTA test days, one trial per day. Groups NNS and SSN were tested for CTA to their familiarized CS $(n=8)$ or to their novel CS $(n=8)$. Groups WWN and WWS $(n=8)$ were tested for CTA only to their novel CSs, saline or saccharin. The measure of performance was amount drunk.

\section{Experiment 2b: Neophobia}

Seventy rats received habituation, pretraining, and aversion conditioning as in Experiment 2a. Groups NNS and NNW ( $n=10$ each) received pretraining with saline $(N)$ and serial conditioning with, respectively, familiarized saline $(N)$ and novel saccharin (S) or familiarized saline (N) and distilled water (W). Groups SSN and SSW ( $n=10$ each) received pretraining with saccharin (S) and conditioning with, respectively, familiarized saccharin (S) and novel saline (N) or familiarized saccharin (S) and water (W). Groups WWN and WWS ( $n=10$ each) received pretraining with distilled water (W) and conditioning with, respectively, water (W) and novel saline (N) or water (W) and novel saccharin (S). So that we could evaluate the effect of the ingestion-toxin pairing, the remaining 10 rats (Group WWW) received pretraining access to water $(W)$ and then water was presented twice (WW) prior to the toxin. Day 7 was recovery day. Days 8-10 were neophobia test days with 3\% casein. Procedures followed those of the preceding experiments.

\section{Experiment 2a: CTA}

\section{Results}

Pretraining and injection-day intakes. In the morning access period of the last pretraining day, Day 5, mean intakes of saline, saccharin, and distilled water were $6.0 \mathrm{~g}, 12.2 \mathrm{~g}$, and $11.0 \mathrm{~g}$, respectively. Saline intake was reliably $(p<.05)$ less than saccharin and distilled water intakes, which did not differ reliably. ANOVA of mean intakes on injection day showed no reliable differences between CSs within a serial-compound pair, for a specific CS flavor (e.g., saline) across groups, or for order of CS presentation. Order of CS presentation was not a reliable factor in any ANOVA of test trial data.

Serial aversion conditioning. Figure 2 presents mean intakes of saline and saccharin CSs in testing. For saline testing, mean intakes by the Pre $S$ and Pre $W$ groups were similar to each other and were less than mean intake for the Pre $\mathbf{N}$ group. For saccharin testing, mean intakes by the Pre N and Pre W groups were similar to each other and were less than mean intake for the Pre $S$ group. Thus, intake of saline or saccharin was less when that flavor was the novel CS, rather than the familiarized CS, in serial aversion training. Intakes increased over test trials for all groups. An ANOVA over the intakes of saline and saccharin, separately, revealed reliable effects for pretraining fluid and test trials [for saline, $F(2,21)=27.85$ and $F(2,42)=31.04$, respectively, $p<.001$; for saccharin, $F(2,21)=25.76$ and $F(2,42)=27.29$, respectively, 


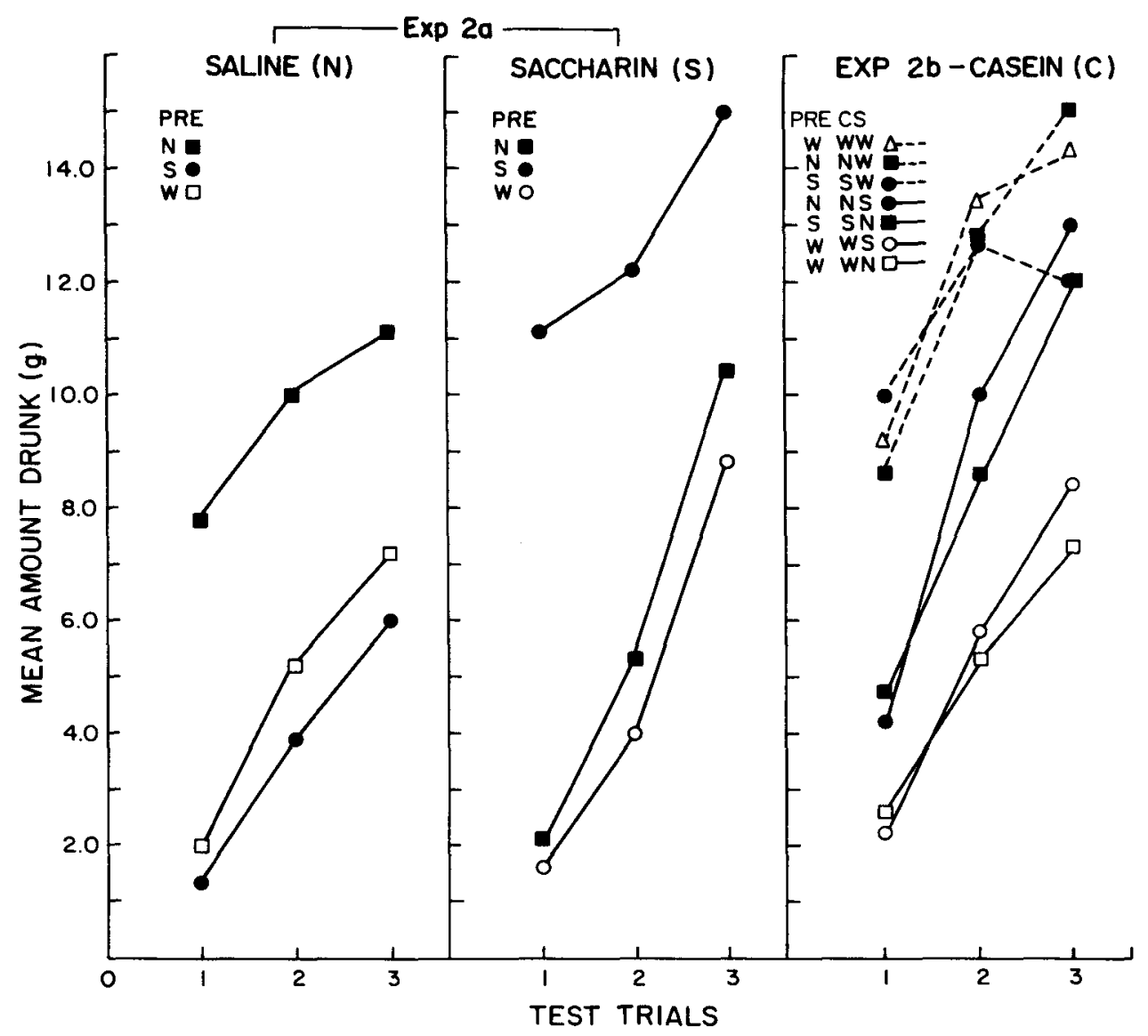

Figure 2. For Experiment (Exp) 2a: Mean amount (in grams) of saline (N) or saccharin (S) drunk on Test Trials 1 -3 after pretraining (PRE) access to saline $(N)$, saccharin (S), or distilled water (W) and serial aversion training with saline and saccharin. For Experiment (Exp) 2b: Mean amount of casein (C) drunk following pretraining (PRE) access to saline (N), saccharin (S), or distilled water $(W)$ and serial aversion training with CS pairs of the pretraining flavor and either of the other two flavors, or water presented twice (WW).

$p<.001$ ], but not for their interaction $(F<1)$. For saline intake, the Pre $\mathrm{S}$ and Pre $\mathrm{W}$ groups each differed from the Pre $\mathrm{N}$ group $(p<.001)$ but not from each other $(t<1)$. For saccharin intake, the Pre $\mathrm{N}$ and Pre W groups each differed from the Pre $S$ group $(p<.001$ but not from each other $(t<1)$.

\section{Experiment 2b: Neophobia}

Pretraining and injection-day intake. On the morning of pretraining Day 5, mean intakes of saline, saccharin, and distilled water were $6.9 \mathrm{~g}, 12.3 \mathrm{~g}$, and $10.0 \mathrm{~g}$, respectively. Only saline intake differed reliably $(p<.05)$ from the other intakes. ANOVA of mean intakes on the injection day revealed no reliable effects between CSs within a serial-compound pair, for a specific CS (e.g., saline) across groups, or for order of CS presentation. The order effect was also not reliable in ANOVA of test trial data.

Postconditioning neophobia. Figure 2 presents mean casein intake during testing for each pretraining fluid and compound CS. Test Trials 1 and 2 show lower casein in- take for groups conditioned with the compound of a novel and a familiarized CS or the compound of a novel CS and distilled water than for groups conditioned with a compound of a familiarized CS and distilled water or a compound of distilled water presentations only. That is, casein intake was less for Groups SSN and WWN, which received conditioning with the compound that included the novel saline CS, than for Groups NNW and WWW. Casein intake was less for Groups NNS and WWS, which received conditioning with the compound that included the novel saccharin CS, than for Groups SSW and WWW. More importantly, casein intake was greater for novel CS groups when preconditioning access occurred to a distinctive novel flavor than to distilled water. Group SSN drank more casein than did Group WWN; Group NNS drank more than did Group WWS.

An ANOVA of all the data for Groups SSN, WWN, and NNW yielded reliable effects for pretraining fluid and test trials $[F(2,27)=20.10$ and $F(2,54)=65.99$, respectively, $p<.001]$, but not for their interaction $(F<1)$. ANOVA and subsequent Scheffé tests for Trial 1 and 
Trial 2, separately, revealed reliable differences for all comparisons $(p<.05)$. ANOVA of all the data for Groups NNS, WWS, and SSW yielded reliable effects for pretraining fluid, test trials, and the interaction of the two $[F(2,27)=29.38, F(2,54)=66.64$, and $F(2,54)=$ 8.19 , respectively, $p s<.001]$. ANOVA and subsequent Scheffé tests for Trial 1 and Trial 2, separately, revealed reliable differences between Groups NNS and WWS on each trial and between each of these groups and Group SSW on Trial $1(p<.001)$. On Trial 2, Groups WWS and SSW differed reliably $(p<.01)$, but Groups NNS and SSW did not. To compare performance for Groups NNW and SSW with that of the ingestion-toxin group, WWW, we applied ANOVA to their casein intakes. Results revealed no reliable effects for groups or groups $\times$ test trials $(F \mathrm{~s}<1)$.

\section{Discussion}

The data from Experiments $2 a$ and $2 b$ provide reliable evidence for the separability of CTA and neophobia. In Experiment 2a, pretraining access to a distinctive novel flavor and pretraining with water yielded similar CTA effects to the novel (saline or saccharin) CS. In Experiment $2 b$, conditioning with a familiar and a novel CS yielded reliably less casein intake than did ingestion-toxin procedures. That is, relative to Group WWW, conditioning-enhanced neophobia occurred for novel-CS groups SSN, WWN, NNS, and WWS. More importantly, for Groups SSN and NNS, pretraining access to a distinctive novel flavor yielded reliably greater casein intake (less neophobia) than did pretraining with water for Groups WWN and WWS. The results of Experiments $2 a$ and $2 b$, together, argue for the separability of CTA from neophobia, because they show that preexposure procedures that failed to alter CTA effects did reliably reduce the demonstration of postconditioning neophobia.

In Experiment 2a, unlike Experiment 1a, the similarity of CTA effects to the novel CS for groups preexposed to water and those preexposed to a novel flavor (not the novel CS) cannot be attributed to a floor effect in conditioning. In Experiment 2a, intake of the novel CS increased reliably over test trials, indicating extinction of CTA and thereby precluding the likelihood of a floor effect.

Furthermore, the results for postconditioning neophobia in Experiment 2b do not seem to be attributable to the flavor-specific properties of saline or saccharin, or to stimulus generalization effects based on the similarity or commonality of flavor characteristics (e.g., taste) among saline, saccharin, and casein. Postconditioning neophobia to casein was similar following conditioning with novel saline and novel saccharin for Groups WWN and WWS. Postconditioning neophobia was similarly reduced by preexposure to a novel flavor prior to conditioning with another novel flavor for Groups SSN and NNS. There were also no reliable differences in casein intake for saline- and saccharin-preexposed groups NNW and SSW, which received ingestion-toxin procedures. Thus, evidence for postconditioning neophobia in Experi- ment $2 b$ seems unrelated to the specific flavor characteristics of saline and saccharin, because similar effects occurred regardless of which flavor, saline or saccharin, was the preexposed flavor or the novel CS. Experiment 2b's results were also unlikely to be due to flavor stimulus generalization among saline, saccharin, and casein. Experiment 2a showed that saline and saccharin flavors were sufficiently dissimilar that preexposure to one of them did not affect CTA to the other. Consequently, it seems untenable to argue that these flavors would both be sufficiently similar to casein to allow for comparable generalization effects from preexposure and/or conditioning, and thereby to occasion the similarity of neophobia effects shown by Groups SSN and NNS and by Groups WWN and WWS. (Best and Batson [1977, p. 141] made a similar argument regarding neophobia to two dissimilar flavors, vinegar and casein, following conditioning with a single CS, coffee.) Furthermore, in conditioning for Groups SSN and NNS, the familiarized CS (saccharin) in one training sequence (SSN) was the novel CS in the other (NNS), and vice versa. If postconditioning neophobia effects were based on stimulus generalization of flavor characteristics from saline and saccharin to casein, generalization should occur regardless of which flavor, saline or saccharin, was the familiar or the novel CS. Generalization of aversion or avoidance tendencies should occur from a novel CS to casein. Generalization of appetitive or approach tendencies should occur from a familiar CS to casein. Because saccharin and saline occurred as the familiar and the novel CS, respectively, in serial conditioning for Group SSN and vice versa for Group NNS, it seems gratuitous to attribute the finding of similar reliable increases in postconditioning neophobia for these groups to flavor generalization from saline and saccharin to casein. Conceivably, generalization effects from the familiar and the novel CS in each training sequence could have cancelled each other and yielded a null effect for conditioning-enhanced neophobia.

\section{GENERAL DISCUSSION}

Our experiments showed that prior ingestion of a novel flavor with impunity yielded effects that survived the establishment of aversion to a different novel flavor and subsequently influenced the acceptability of other novel flavors. Best (1975, Experiment 4A) reported a similar finding in studies of conditioned and latent inhibition. In Experiments $1 b$ and 2b, prior "safe" ingestion of novel saline or saccharin reduced the demonstration of conditioning-enhanced neophobia to casein hydrolysate. Because this effect occurred without apparent diminution of CTA to the novel CS, it appears that prior ingestion history affects neophobia through a process which differs from that by which prior ingestion experience affects CTA (see Braveman \& Jarvis, 1978, and Miller \& Holzman 1981).

In postconditioning neophobia, prior acquisition of CTA presumably augments the aversiveness or emotionality occasioned by the novel flavor in neophobia testing. Prior 
ingestion of a distinctive novel flavor with impunity may curtail the aversiveness of novelty, and thereby attenuate one basis for postconditioning neophobia. In Experiments $\mathrm{lb}$ and $2 \mathrm{~b}$, prior ingestion of novel saline or saccharin flavors may have reduced the aversiveness of novelty. Consequently, in neophobia testing, novel casein may have occasioned less aversiveness or emotionality to be influenced by prior conditioning procedures.

Experiment $2 \mathrm{~b}$ showed that postconditioning neophobia was reduced whether saline or saccharin was the preexposure flavor. These results support the view that novelty may reflect a general process that is not necessarily related to the flavor-specific properties (e.g., taste or intensity) of the stimuli used (Miller \& Holzman, 1981). The nonspecific character of novelty conceivably may have permitted the influence of novel flavor preexposure to endure the acquisition of CTA. If CTA occurred to the specific characteristic of a flavor and novelty has no unique stimulus properties of its own (Miller \& Holz man, 1981, p. 100), then establishing an aversion to a particular flavor may not affect responding that is related to the novelty of another flavor.

The present results provide further support for an associative analysis of conditioning-enhanced neophobia (Domjan, 1980; Franchina \& Fitzgerald, 1983). Consistent with an associative viewpoint, Experiments $1 \mathrm{~b}$ and $2 b$ showed that (1) the demonstration of postconditioning neophobia depended upon novel flavor-toxin pairing; (2) procedures that reduced CTA, such as preconditioning access to the CS, yielded negligible evidence of enhanced neophobia; and (3) the selective associability of a novel flavor and a toxin in serial aversion conditioning yielded reliable enhancement of neophobia. However, Experiments $1 b$ and $2 b$ showed that enhanced neophobia was not an invariant consequence of novel flavor-toxin pairing. The amount of postconditioning enhancement may depend upon the rat's preconditioning history of ingestion. If previous ingestion of novel flavors occurred without negative consequences, the impact of aversion conditioning on subsequent ingestion of other novel flavors may be reduced.

Finally, in view of the ubiquitous diet of omnivores such as the rat, the separability of neophobia and CTA seems a reasonable, if not a fortuitous, circumstance. If neophobia were to increase as an invariant consequence of aversion conditioning, the rat's prior history of nonaversive contact with novel flavors or edibles might be nullified. If that happened, the rat's wide food selection could be constrained and, as familiar food became less available in the environment, the organism's capability for survival might be in jeopardy (Barnett \& Cowan, 1976).

\section{REFERENCES}

Ahlers, R. H., \& BeST, P. J. (1971). Novelty vs. temporal contiguity in learned taste aversions. Psychonomic Science, 25, 34-36.

BARNeTt, S. A., \& CowAN, P. E. (1976). Activity, exploration, curiosity and fear: An ethological study. Interdisciplinary Science Reviews, $1,43-62$.

BEST, M. R. (1975). Conditioned and latent inhibition in taste-aversion learning: Clarifying the role of learned safety. Joumal of Experimental Psychology: Animal Behavior Processes, 104, 97-113.

BEst, M. R. \& BATSON, J. D. (1977). Enhancing the expression of flavor neophobia: Some effects of the ingestion-illness contingency. Journal of Experimental Psychology: Animal Behavior Processes, 3, 132-143.

Braveman, N. S., \& JARVIS, P. S. (1978). Independence of neophobia and taste aversion learning. Animal Learning \& Behavior, 6, 406-412.

Carroll, M. E., Dinc, H. I., Levy, C. J., \& SMiTh, J. C. (1975) Demonstrations of neophobia and enhanced neophobia in the albino rat. Joumal of Comparative \& Physiological Psychology, 89, 457-467.

Domjan, M. (1976). Deterninants of the enhancernent of flavored-water intake by prior exposure. Journal of Experimental Psychology: Animal Behavior Processes, 2, 17-27.

DOMJAN, M. (1980). Ingestional aversion learning: Unique and general processes. In J. S. Rosenblatt, R. A. Hinde, C. Beer, \& M. C. Busnel (Eds.), Advances in the study of behavior (Vol. 2). New York: Academic Press.

Franchina, J. J., \& Fitzgerald, B. A. (1983). Poison-enhanced neophobia: Effects of CS-UCS/UCS-CS procedures and long-delay training. Learning \& Motivation, 14, 351-366.

Franchina, J. J., Silber, S., May, B. (1981). Novelty and temporal contiguity in taste aversion learning: Within-subjects conditioning effects. Bulletin of the Psychonomic Society, 18, 99-102.

Krane, R. V., Sinnamon, H. M., \& Thomas, G. J. (1976). Conditioned taste aversions and neophobia in rats with hippocampal lesions. Journal of Comparative \& Physiological Psychology, 90, 680-693.

Milleg, R. R., Holzman, A. D. (1981). Neophobias and conditioned taste aversions in rats following exposure to novel flavors. Animal Learning \& Behavior, 9, 89-100.

Mitchell, D., Winter, W., \& Moffitt, T. (1980). Cross-modality transfer: Exteroceptive context habituation enhances taste neophobia and conditioned taste aversions. Animal Learning \& Behavior, 8, 524-528.

ReVusky, S. H., \& BeDARF, E. W. (1967). Association of illness with prior ingestion of novel food. Science, 155, 219-220.

SIEGEL, S. (1974). Flavor preexposure and "learned safety." Journal of Comparative \& Physiological Psychology, 87, 1073-1082.

TARPY, R. M., \& McINTosh, S. M. (1977). Generalized latent inhibition in taste aversion learning. Bulletin of the Psychonomic Society, 10, 379-381.

(Manuscript received April 10, 1985; revision accepted for publication April 9, 1986.) 